Théologiques

Théologiques

\title{
Gestes admirables ou le langage saisissant du corps dans la poétique de Friedrich Schiller
}

\section{Monique Moser-Verrey}

Volume 17, numéro 1, 2009

Les charismes

URI : https://id.erudit.org/iderudit/039498ar

DOI : https://doi.org/10.7202/039498ar

Aller au sommaire du numéro

Éditeur(s)

Faculté de théologie et de sciences des religions, Université de Montréal

ISSN

1188-7109 (imprimé)

1492-1413 (numérique)

Découvrir la revue

Citer cet article

Moser-Verrey, M. (2009). Gestes admirables ou le langage saisissant du corps dans la poétique de Friedrich Schiller. Théologiques, 17(1), 51-77.

https://doi.org/10.7202/039498ar
Résumé de l'article

Le charisme en question est abordé par le biais de sa représentation dans $L e$ camp de Wallenstein, pièce construisant par l'échange d'opinions le pouvoir extraordinaire d'un leader absent. Ce paradoxe illustre à merveille le travail du poète qui, par ses mots, donne à voir des corps vivants et des gestes admirables. L'analyse des figures du corps parlant porte sur le corpus des nouvelles de Schiller et révèle sa maîtrise de l'écriture sensible en vogue dans l'Europe des Lumières. Elle montre aussi son souci de l'unité classique et sa recherche du beau geste critique des intrigues courtisanes et de l'absolutisme arbitraire. 


\title{
Gestes admirables ou le langage saisissant du corps dans la poétique de Friedrich Schiller
}

\author{
Monique MOSER-VERREY* \\ Littératures et langues modernes \\ Université de Montréal
}

\section{Le charisme de Wallenstein et sa représentation}

Ernst ist das Leben, heiter ist die Kunst.

La vie est sérieuse, l'art est enjoué1. (Schiller 1975b, 274)

Ce vers célèbre, qui oppose le sérieux de la vie à la gaieté de l'art, sert de point final au prologue du Camp de Wallenstein, le premier volet du chefd'œuvre de Schiller intitulé Wallenstein. Ein dramatisches Gedicht. Cette trilogie est entièrement consacrée au chef de guerre charismatique par excellence que fut Albrecht Wenzel Eusebius von Wallenstein duc de Friedland qui fit carrière au cours de la guerre de Trente Ans dans l'espoir de devenir roi de Bohème. Dans ce prologue (Schiller 1975b, 270-274), prononcé en octobre 1798 à Weimar lors de la réouverture du théâtre Schaubühne, le poète expose son sujet dont il souligne l'actualité. En effet, secouée par la Révolution française et les guerres qui s'ensuivent, l'Europe voit alors s'effriter le cadre politique des empires chèrement rétabli cent cinquante ans auparavant lors des traités de Westphalie en 1648 qui avaient mis fin à trente années de guerres et de misères. Ce fonds sombre et lamentable est le décor dans lequel Schiller choisit de faire briller le caractère dévoyé

* Monique Moser-Verrey est professeur titulaire au Département de littératures et de langues modernes de l'Université de Montréal. Elle anime le groupe de recherche sur les Lumières allemandes et européenne au Centre canadien d'études allemandes et européennes. Sa recherche porte actuellement sur les livres à gravures des années 1760 à 1800. En septembre 2009, elle a fait paraître aux PUL en collaboration avec Lucie Desjardins et Chantal Turbide Le corps romanesque. Images et usages topiques sous l'Ancien Régime.

1. Sauf avis contraire, les traductions sont de l'auteur. 
(verwegener Charakter) mais extraordinaire d'un prince qui fut à la fois le soutien et la terreur de son empereur. Comment put-il lever à ses frais une armée plus redoutable que celle du Saint Empire puis, allant de gloire en gloire, oser trahir son seigneur pour faire face enfin en 1634 à une mort infamante? Selon l'avis du poète, c'est le camp qui explique son crime: «Das Lager nur erkläret sein Verbrechen».

Pour montrer comment son héros a pu être séduit par le pouvoir au point de recourir à une conjuration ${ }^{2}$ pour satisfaire ses ambitions, Schiller réussit le tour de force d'évoquer Wallenstein tout au long de la première pièce de la trilogie qui lui est consacrée sans le faire entrer en scène une seule fois. Il n'apparaîtra sur scène que dans les deux pièces suivantes, Les Piccolomini et La mort de Wallenstein. C'est la rumeur qui porte aux nues le leader charismatique et prête au chef de guerre un courage extraordinaire ainsi que des pouvoirs hors du commun. Si des soldats venus des quatre coins du Saint Empire croient en Wallenstein, veulent le suivre et sont prêts à donner leur vie dans cette aventure, comme ils le chantent en chœur à la fin du spectacle, c'est que la chance est de son côté. Il remporte toutes les victoires et à la bataille de Lützen, qu'il a pourtant perdue mais où son adversaire Gustave-Adolf, roi de Suède, a trouvé la mort, il a posé un geste admirable donnant à voir son corps invulnérable.

Denn in der blutgen Affär bei Lützen

Ritt er euch unter des Feuers Blizen

Auf und nieder mit kühlem Blut.

Dans la sanglante affaire de Lutzen, au milieu des éclairs du feu de l'ennemi, il chevauchait de-ci et de-là avec un parfait sang-froid. (Schiller 1975b, 288)

Les balles avaient beau percer son chapeau, son manteau et ses bottes, elles n'entamaient pas sa peau. Certains prétendent qu'un baume magique protège son corps, qu'il sait lire l'avenir dans les étoiles, bref, qu'il est en lien avec le diable, mais d'autres en doutent (1975b, 288). Quoi qu'il en soit, Wallenstein est présent dans toutes les histoires qui se racontent au camp de Pilsen, peu avant son assassinat. Il est une figure marquante, déjà faite de mots avant sa disparition, ein Schattenbild dit le poète: in einer Reihe von Gemälden (1975b, 273). Cette ombre chinoise et ces tableaux, rendus en vers rimés à l'allemande, transportent la vérité bien sombre du sujet dans le monde ludique et factice de l'art. Le poète invite enfin son public à la

2. Réfractaire à l'autorité des princes, Schiller s'est beaucoup intéressé aux conjurations dans l'histoire (voir Moser-Verrey 2006, 325-339). 
reconnaissance car l'illusion que crée la poésie se résorbe d'elle-même et ne substitue pas son apparence à la vérité ( «ihren Schein / Der Wahrheit nicht betrüglich unterschiebt») (1975b, 274). Ce sont les modalités qui diffèrent, puisque la vie, on l'a vu, est sérieuse, mais la poésie joyeuse.

Les questions que soulèvent Le camp de Wallenstein et son prologue sont au cœur de l'énigme du charisme, des gestes admirables qui soutiennent sa crédibilité et de la représentation de ces phénomènes qui contribue à orienter l'action sociale et politique. Schiller n'est pas un intervenant naïf dans ce débat. Dès son plus jeune âge il est acclamé en tant que dramaturge révolutionnaire. Sa pièce intitulée Les brigands soulève en 1782 un enthousiasme qui se compare au choc provoqué huit ans plus tôt par la publication du Werther de Goethe. Ces deux œuvres provocatrices participent d'un bref mouvement littéraire appelé le Sturm und Drang, assaut impulsif de la jeune génération contre les normes et les politiques d'une société régie par l'absolutisme des princes. Schiller échappe lui-même à l'emprise du duc Charles-Eugène de Wurtemberg pour se tailler une place dans la vie des lettres en toute liberté à la faveur de publications périodiques, de travaux pour le théâtre et d'entreprises éditoriales. Son établissement comme professeur d'histoire à l'Université d'Iéna n'aura lieu qu'en 1789 après la publication de l'Histoire du soulèvement des Pays-Bas, une matière déjà travaillée dans Don Carlos Infant von Spanien. Ein dramatisches Gedicht. Si je cite ici le titre original, c'est pour rappeler qu'il est double et situe l'œuvre dans le cadre classique selon lequel l'art du poète peut emprunter les trois voies que sont la poésie lyrique, la poésie dramatique et la poésie épique.

Dans le cas du Wallenstein, le traité d'histoire précède l'élaboration du drame historique. Au début des années 1790, Schiller avait, en effet, publié son Histoire de la guerre de Trente Ans qui souligne déjà le destin étonnant de Wallenstein. Il y remarque, entre autres, le double assassinat de ce prince singulier qui paya son audace de sa vie, puis fut très mal jugé par une historiographie sortie de la plume de ses ennemis car ils avaient tout intérêt à anéantir et à occulter ses projets de paix et de royaume débarrassé des

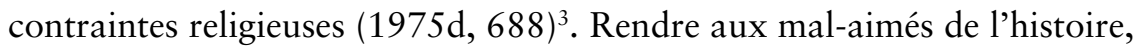
épris de liberté, une dimension humaine qui touche, discerner dans leurs actions le sublime qui seul confère au pathos une dimension esthétique (Schiller 1998, 7) et orienter par ce moyen la conduite morale et politique de ses contemporains, voilà ce que recherche alors Schiller. Il contribue à doter la scène allemande d'un théâtre classique en étroite collaboration

3. Voir aussi la documentation conséquente réunie dans Rothmann (1999). 
avec Goethe et réfléchit aux voies qui s'ouvrent à l'esthétique en s'appropriant et en développant la pensée kantienne exposée dans la Critique de la faculté de juger. Pour pouvoir représenter la beauté du geste ${ }^{4}$ qui retient l'attention générale, Schiller tient compte de la sensibilité naturelle aux êtres humains, de la volonté raisonnée qui fait façon de leurs désirs et de leur douleur, mais aussi des égards dus à la communauté. Dans ce sens, son message est davantage critique que révolutionnaire. La vérité historique lui sert de cadre, mais la vérité poétique est le but ultime de ses pièces conçues pour le délassement et le plaisir du public, même lorsque le sujet est sombre, voire tragique.

L'articulation d'une théorie esthétique de même que la maîtrise pratique, qui s'observe dans les pièces historiques qu'il a données au théâtre allemand pour une scène se voulant alors nationale, témoignent d'un vif intérêt pour la poétique. En fait, Schiller n'a pas cessé d'être attentif à l'évolution de l'art poétique et aux voies qu'empruntait dans l'Europe du XVIII ${ }^{\mathrm{e}}$ siècle une nouvelle écriture de la sensibilité, se donnant pour objet la nature humaine en général. La critique a consacré de nombreuses pages à sa poésie lyrique ainsi qu'à son théâtre, mais elle a beaucoup moins fréquenté son écriture romanesque. Pourtant, Schiller a aussi exploré les possibles de la figuration des corps sensibles et de leurs gestes admirables dans le cadre de l'anecdote, du récit bref, de la nouvelle ou du petit roman. Mon analyse de la façon dont il travaille la représentation narrée du corps des personnages, appelés à susciter l'admiration des lecteurs par leurs gestes, se limitera maintenant au corpus moins travaillé de ses nouvelles.

Mais avant d'aborder l'analyse, il importe de préciser que le geste admirable n'est pas forcément exemplaire. Il peut également être effrayant et retenir l'attention à ce titre. Comme l'établit Descartes dans son Traité des passions de l'âme, l'admiration est la première des quarante passions qu'il y dénombre. Cette passion naît de la surprise que cause un objet inconnu qui semble rare ou extraordinaire avant même que l'on sache s'il est bon ou non. Elle ne peut donc pas avoir son contraire puisqu'un objet connu ne suscite pas la surprise et l'étonnement. Tout en étant avec l'amour, la haine, le désir, la joie et la tristesse l'une des six passions primitives analysées dans le Traité, l'admiration diffère des autres passions dans la mesure où elle engage le cerveau plutôt que le cœur. Elle permet d'acquérir de nouvelles connaissances et de les conserver, parce que l'attention se porte avec force

4. Nicolas Briand intitule précisément son introduction aux Textes esthétiques de Schiller «Pour la beauté du geste». 
vers l'impression nouvelle. Ainsi l'admiration fait apprendre de nouvelles choses. Elle est nécessaire à tous ceux qui ne veulent pas demeurer ignorants. Cette analyse, contenue dans la seconde partie du Traité aux articles 53, et 70 à 73, valorise l'aspect dégagé de l'admiration qui «n'a point de rapport avec le cœur et le sang, desquels dépend tout le bien du corps » (Descartes 1953, 728), et qui donne de la force à toutes les autres passions quand elle est jointe à celles-ci. L'admiration tire son emprise «de la nouveauté, et de ce que le mouvement qu'elle cause a dès son commencement toute sa force» $(1953,729)$. Ces remarques nous montrent qu'il n'y a pas de demi-mesure en ce qui a trait à l'admiration. Elle entraîne avec force le sujet vers ce qui lui semble extraordinaire. C'est afin d'éveiller cette passion pour son personnage dans l'âme de son lecteur que le poète cherche à rendre la représentation du corps de celui-ci saisissante.

\section{Les nouvelles de Schiller et le roman sensible}

Le temps que Schiller a consacré à écrire des nouvelles (1782-1789) est réputé constituer un «intermède " $(1975$ e, 1057) à l'intérieur de son œuvre d'écrivain et il semble accorder lui-même peu d'importance à cette activité coupable ( «Eines sündlichen Zeitaufwands », Lettre à Körner du 17 mars 1788 citée par Ebnet 1993, 13). Dans un effort de réhabiliter les nouvelles de Schiller, Emil Staiger distingue nettement celles qui ne constituent qu'une ébauche, comme «Eine großmütige Handlung, aus der neusten Gechichte », un simple travail de rédaction à partir d'un matériau trouvé ailleurs, comme «Herzog von Alba bei einem Frühstück auf dem Schlosse zu Rudolstadt. Im Jahre 1547 », ou encore la mise en forme de récits oraux, comme «Spiel des Schicksals", des deux textes qui sortent du lot: "Der Verbrecher aus verlorener Ehre » et "Der Geisterseher» (1964, 269-272). L'une et l'autre de ces narrations ont bénéficié chez José Corti d'une édition française intéressante et largement documentée. Ainsi la tradition française inscrit Criminel par infamie. Une histoire véritable, traduit et présenté par René Radrizzani (1990) et Le visionnaire, traduit par Albert Béguin et commenté par Pierre Péju (1996) dans une "Collection romantique » choisissant de présenter le volet romanesque ou plutôt narratif de l'œuvre schillérienne en rapport avec sa philosophie de l'histoire (Radrizzani 1990, 90) ou comme l'expression d'une double inquiétude relative, d'une part, au pouvoir quasi magique de l'œuvre théâtrale — que l'on pense au retentissement de la première représentation des Brigands - puis, d'autre part, au théâtre tout intérieur des influences qui rendent l'individu raisonnable et plein de bonne volonté 
néanmoins perméable à l'irrationnel et aux pratiques ésotériques. Cette préoccupation toute romantique rejoint, en effet, certains contes de Hoffmann (Péju 1996, 10-11). L'auteur classique, Schiller, se révélerait ainsi secrètement romantique et même moderne si l'on tient compte de la forme fragmentaire, trouble et incertaine du Visionnaire (Staiger 1964, 284; Péju 1996, 9).

Le statut ambigu et même exploratoire des nouvelles de Schiller se reflète d'ailleurs dans des regroupements variables d'une édition à l'autre. Tandis que les Euvres complètes rangent "Herzog von Alba bei einem Frühstück auf dem Schlosse zu Rudolstadt. Im Jahre 1547 » parmi les textes d'histoire, cette " anecdote " (Schiller 1975d, 988) est incluse dans les éditions séparées des nouvelles déjà citées. Par contre, ces éditions excluent la traduction de l'épisode de Madame de la Pommeraye que Schiller emprunte à Jacques le fataliste de Diderot et publie, en 1785, dans le premier cahier de sa revue Thalia sous le titre "Merkwürdiges Beispiel einer Weiblichen Rache». Cette traduction assez libre figure bien parmi ses nouvelles dans les Euvres complètes.

En fait, Schiller puise à bien des sources, comme c'était d'ailleurs l'usage, pour nourrir sa plume de nouvelliste et il n'est pas impertinent de remarquer qu'il convoque dès l'abord les grands maitres du roman sensible, soit Richardson et Diderot, afin de découvrir à son tour les astuces de la fiction vraie. Contrairement à Diderot, Schiller ne fait pas l'éloge de Richardson. Voilà ce qu'il en dit dans «Eine großmütige Handlung, aus der neusten Gechichte », sa première nouvelle parue dès 1782:

Gegenwärtige Anekdote von zween Teutschen - mit stolzer Freude schreib ich das nieder - hat ein unabstreitbares Verdienst - sie ist wahr. Ich hoffe, daß sie meine Leser wärmer zurücklassen werde als alle Bände des «Grandison» und der «Pamela».

La présente anecdote de deux Allemands - j'écris cela avec joie et fierté - possède un mérite incontestable - elle est vraie. J'espère qu'elle donnera à mes lecteurs plus de chaleur que ne le feraient tous les volumes du Grandison ou de Paméla. (Schiller 1975e, 9)

Ce qui doit distinguer l'anecdote allemande des spectacles et des romans à la mode, c'est son authenticité censée garantir chez les lecteurs une chaleur qui les portera vers la bienfaisance, si l'on en croit le paragraphe précédent. Car il y est supposé que les larmes versées sur la bonté d'un être fictif ferment les cœurs aux besoins du prochain, de l'être humain en chair et en os.

Cette entrée en matière renverse l'argumentation qu'offre Diderot dans son Éloge de Richardson. Il y est bien évidemment aussi question de vérité, 
mais celle-ci ne doit justement pas être anecdotique. Elle doit bien au contraire rendre justice à la nature humaine en général:

Ô Richardson! j'oserai dire que l'histoire la plus vraie est pleine de mensonges, et que ton roman est plein de vérités. L'histoire peint quelques individus: tu peins l'espèce humaine [...]. Ô peintre de la nature! c'est toi qui ne mens jamais. (Diderot 1996, 162-163)

Par le fait même, tous les lecteurs peuvent se reconnaître et s'enflammer à la lecture de ses romans. Dès l'abord, Diderot note qu'on "prend, malgré qu'on en ait, un rôle" $(1996,155)$ dans les œuvres de Richardson et qu'on y apprend à être bon et juste, car «Richardson sème dans les cœurs des germes de vertus» $(1996,156)$. Cette semence fait son œuvre peu à peu et lorsque l'occasion s'en présente "on se sent porter au bien avec une impétuosité qu'on ne se connaissait pas» $(1996,156)$. Schiller ne conçoit pas cet effet différé du modèle romanesque et prétend que le lecteur peut s'émouvoir d'une fiction et agir durement dans la réalité tout à la fois («In dem nämlichen Augenblick» $(1975$ e, 9)). Il se méfie de l'art du roman qui fait surgir une existence artificielle dans un monde d'idées ("gekünstelte Existenz in einer idealischen Welt» $(1975 \mathrm{e}, 9))$. Bien au contraire, Diderot s'extasie devant l'habileté de Richardson à créer l'illusion par une multitude de petites choses difficiles à imaginer et à rendre. Il évoque ici le «sublime» du geste et du mot et les "vérités de détail qui préparent l'âme aux impressions fortes des grands événements » et permettent finalement d'y adhérer (1996, 159-160). Comme on le sait, Schiller ne restera pas étranger aux idées qu'avance Diderot dans son Éloge de Richardson, mais il développera ce que Pierre Hartmann appelle "une dramaturgie du sublime» $(1997,79-170)$ après la Révolution française.

Ce qui permet aux lecteurs de «connaitre» Paméla, Clarisse et Grandison et de se faire "une image des personnages que l'auteur a mis en scène " (Diderot 1996, 161), c'est l'évocation de leurs physionomies, de leurs coiffures, de leurs postures, de leurs mines, de leurs gestes, de leurs regards, de leurs larmes et leurs soupirs, en un mot: de leurs corps sensibles. Selon Diderot, l'élégante traduction française de l'abbé Prévost prive les lecteurs des ingrédients nécessaires à l'empathie, puisque le traducteur abrège les passages les plus pathétiques où l'on voit, par exemple, miss Howe «échevelée et étendue sur le cercueil de son amie [Clarisse], se tordant les bras, levant ses yeux noyés de larmes vers le ciel» (1996, 160). Quoi qu'il dise de son incapacité à lire Clarisse pour se former, tant "[l]'intérêt et le charme de l'ouvrage dérobent l'art de Richardson» (1996, 163), il est certain que 
l'écriture et la dramaturgie de Diderot s'inspirent entre autres du « roman domestique» de Richardson, comme le démontre Henri Lafon dans son étude sur les liens entre le roman et le drame dans la seconde moitié du XVIII ${ }^{\mathrm{e}}$ siècle (2001, 440-441). C'est la pantomime qui fait voir le personnage et affecte les cœurs. Elle le livre corps et âme au lecteur-spectateur.

Si Diderot se forme à la lecture de Richardson, Schiller se forme, pour sa part, à la lecture de Diderot. Sa traduction de l'épisode de Madame de la Pommeraye témoigne même d'une lecture particulièrement attentive. Son éloge de Diderot est plus bref que celui que Diderot consacre à Richardson, mais il ne manque pas. Après avoir remarqué que la vengeance de Madame de la Pommeraye rebute, bien que Diderot tente de la justifier, il loue "die kühne Neuheit dieser Intrige, die unverkennbare Wahrheit der Schilderung, die schmucklose Eleganz der Beschreibung» (l'audacieuse nouveauté de cette intrigue, la vérité manifeste de la narration et l'élégance dépouillée de la description; Schiller 1975e, 218-219) et doute que sa traduction ait put atteindre les particularités de l'original. L'hommage ainsi offert à un maître coïncide d'ailleurs dans les deux cas avec la disparition de celui-ci. Si Schiller honore Diderot dans le premier cahier de sa revue Thalia en 1785 , c'est que Diderot était mort au cours de l'été 1784 . Pour sa part, Diderot avait publié l'Éloge de Richardson en 1762 tandis que le romancier anglais était décédé l'année précédente. Même si Schiller commence son aventure de nouvelliste par un rejet des romans de Richardson, une certaine filiation éthique et esthétique semble indéniable. Mais, contrairement au vieux Diderot qui admire la façon dont Richardson sait «conduire des drames de trente à quarante personnages» $(1996,163)$, le jeune Schiller n'éprouve pas d'attrait pour les œuvres d'une grande étendue. Dans sa correspondance avec Goethe, il évalue d'ailleurs que son esprit est plus apte à faire fructifier un fonds restreint «et à créer, par le moyen de la forme, la multiplicité variée qui fait défaut au contenu " (Goethe-Schiller 1994a, 52). La même réticence face à l'œuvre très étendue s'aperçoit en outre dans «Der Geisterseher », lorsqu'il est question des Noces de Cana, de Paul Véronèse. Le narrateur note que cette composition comptant cent vingt personnages lui a donné plus de surprise que de véritable plaisir:

Welches menschliche Auge kann ein so zusammengesetztes Ganze erreichen und die ganze Schönheit, die der Künstler darin verschwendet hat, in einem Eindruck geniessen!

Quel regard humain serait en mesure de saisir une totalité aussi complexe et de jouir en un seul coup d'œil de toute la beauté que l'artiste a répandue dans sa composition! (Schiller 1975e, 128) 
Il apparaît enfin que, l'œuvre devant constituer un tout, la chaleur recherchée par Schiller ne serait pas seulement une fonction de la vérité, mais encore le résultat d'une impression unique. Ce besoin qu'a Schiller d'embrasser le tout, en littérature comme en peinture, est particulièrement évident lorsque, après avoir parcouru en deux jours les huit livres de Wilhelm Meister, il écrit à Goethe:

J'avoue qu'à l'heure qu'il est j'en ai bien saisi la continuité, mais non pas encore l'unité; toutefois, je ne doute pas un seul instant que je doive parvenir à l'embrasser, elle aussi, avec une entière clarté, à supposer même que, lorsqu'il s'agit d'œuvres de ce genre, la continuité ne soit pas déjà plus que la moitié de l'unité. (Goethe-Schiller 1994a, 218)

Reste à voir si Schiller a su donner à ses propres nouvelles l'unité qu'il recherche dans le roman de Goethe. Pour apprécier la réalisation de cette exigence, l'analyse portera maintenant sur la représentation du corps des personnages dans ces nouvelles.

\section{Portraits, tableaux et scènes}

Afin de mieux saisir la construction du corps romanesque, il est utile de distinguer quelques sous-genres qui en présentent des aspects signifiants. Sans m'étendre sur une méthode d'analyse que j'ai eu l'occasion de développer et d'éprouver dans d'autres contextes (Moser-Verrey 2001, 349-351), je dirai simplement qu'elle distingue les portraits des tableaux et des scènes. Ce qui est dit du corps des personnages affiche dans le premier cas leur identité, dans le second leurs états d'âme ou leurs pensées et dans le dernier cas leur sociabilité. Ces évocations du corps sont généralement codées et relèvent de figures du descriptif qui se modifient d'une époque à l'autre. Tandis que le sous-genre du portrait était très à la mode en France lors de l'époque classique dans la seconde moitié du XVII siècle, le sous-genre du tableau lui vole la vedette un siècle plus tard. Du côté de la scène, on observe une prise en compte croissante de l'interaction non verbale au cours du XVIII ${ }^{\mathrm{e}}$ siècle au point où des scènes muettes prennent parfois la place de scènes dialoguées. Le roman sensible sera donc gorgé de tableaux et de scènes muettes car de telles mises en évidence des mouvements du corps servent à révéler au lecteur-spectateur l'intériorité des personnages. Richardson et Diderot sont les principaux artisans de cette mise en présence du personnage. Se plaignant de la confusion des genres qu'on observe 
chez les Modernes, Goethe met d'ailleurs le doigt sur la dramatisation de la narration:

On observe de même, lorsqu'on suit la poésie dans son évolution, que tout y tend au drame, c'est-à-dire à la représentation sous la forme de la présence parfaite. C'est ainsi que les romans par lettres ont un caractère pleinement dramatique, et que l'on peut donc à bon droit y insérer des dialogues proprement dits, ainsi que l'a fait Richardson [...]. (Goethe-Schiller 1994a, 501)

Quant à Schiller, ce n'est pas tant la mise en présence des personnages romanesques qu'il reproche à Richardson et à ses imitateurs que le fait «d'en tirer lourdement un parti dramatique, et de dépenser des trésors d'indélicatesse à exploiter la délicatesse [des] sentiments!» (Goethe-Schiller 1994a, 220). La question est de savoir avec quelle délicatesse il met lui-même en scène le corps de ses personnages en tant que nouvelliste. Nous verrons cela en abordant ses nouvelles l'une après l'autre dans l'ordre chronologique de leur parution.

\section{Un geste généreux tiré de l'histoire contemporaine}

«Eine großmütige Handlung, aus der neusten Gechichte» illustre de la façon la plus concise la construction schillérienne d'une totalité. Dans sa brièveté, cette anecdote ne contient que trois portraits et trois tableaux. En effet, elle ne met en scène que trois personnages. Deux frères sont amoureux de la même jeune fille dont voici le portrait physique et moral réduit à sa plus simple expression: «das Fräulein war schön und zur Empfindung geschaffen " (la demoiselle était belle et faite pour la vie sentimentale; Schiller 1975e, 9). Le frère aîné s'en va en Hollande pour tâcher d'oublier la belle, mais son image le poursuit de sorte que le malheureux en tombe malade. Son portrait fait alors pitié: "halbverwest, ein wandelndes Gerippe, das erschröcklichste Bild des zehrenden Kummers " (à moitié décomposé, un squelette ambulant, l'image la plus effrayante d'un corps rongé par le chagrin; 1975e, 10). De retour chez lui, il perd tous ses moyens et se donne en spectacle. Le tableau est le suivant: "ohnmächtig sank er in die Arme des Fräuleins» (Il tomba évanoui dans les bras de la jeune fille; 1975e, 10). Le cadet se jette alors dans une voiture pour céder la place à son malheureux frère. La scène du départ donne lieu à un petit tableau: «halb entseelt starrten ihm die Hinterbleibenden nach » (à moitié privée de son âme, la famille le regardait partir; 1975e, 11). Le voyageur se rend jusqu'en Indonésie d'où il écrit une lettre à son frère, acte généreux dépeint dans un autre petit tableau: «Gott hat mir die Kraft geschenkt, der Freundschaft das 
höchste Opfer zu bringen, dein ist — Gott! hier fiel eine Träne — die letzte — Ich hab überwunden - Dein ist das Fräulein» (Dieu m'a donné la force, d'offrir à l'amitié le plus grand des sacrifices. À toi - Dieu! Une larme tomba - la dernière — J'ai renoncé — La demoiselle est à toi; 1975e, 11).

S'il écrit aussi dans sa lettre que sa blessure saigne toujours, il faut comprendre cette image corporelle au sens figuré. L'anecdote se clôt enfin sur le portrait brillant de cet homme au grand cœur: «der jüngere [...] gediehe zum glücklichen, glänzenden Mann» (le plus jeune [...] devint un homme heureux et brillant; 1975e, 12). Il n'y a rien de superflu dans les portraits et rien de lourd dans les tableaux qui peignent les sentiments en touches légères: une défaillance, un regard, une larme.

Voyons maintenant les symétries qui scellent l'ensemble. Si on analyse les portraits, le vrai couple romanesque se compose de la belle jeune fille décrite au début du récit et du cadet, l'homme brillant, décrit à la fin du récit. Ces beaux corps correspondent à de belles âmes capables de renoncement. Elle avoue sur son lit de mort qu'elle préférait le cadet, tandis que celui-ci fait et tient le vœu du célibat. Quant à l'aîné, il est sans vie à moins d'avoir une épouse à son côté. Il en aura deux, puisque la première meurt au bout d'un an. Les tableaux le montrent sans connaissance et presque sans âme. Sans sa belle il est "halbverwest » et sans son frère " halbentseelt ». Il n'est qu'un demi-corps et une demie-âme. Son entièreté dépend des deux autres personnages qui constituent l'autre moitié de son corps et de son âme. L'ensemble se tient parce que la belle finit par appartenir de corps à l'aîné et de cœur au cadet. Le premier bonheur dure un an selon l'histoire, mais le second est inscrit pour toujours dans la beauté équilibrée du récit qui singularise par leurs portraits les vrais héros.

\section{Exemple curieux d'une vengeance de femme}

Dans son appréciation du rapport de Schiller à Diderot, Benno von Wiese souligne l'admiration qu'éprouvait le premier pour la nouveauté de l'intrigue dans l'histoire de Mme de la Pommeraye (1963, 303). Nous avons vu, cependant, qu'il fait tout autant l'éloge de l'évidente vérité de la narration et de l'élégante sobriété de la description. Dans son étude comparative du texte original de Diderot et de l'adaptation qu'en propose Schiller, Stefan Buck établit à juste titre la préséance de l'intérêt esthétique sur les autres raisons qui peuvent avoir incité Schiller à traduire ce texte de Diderot (1990, 249). Son extraordinaire nouveauté ne concerne pas uniquement l'intrigue, elle concerne surtout la technique narrative toute faite de provocations. 
L'adaptation schillérienne consiste d'abord à donner le statut de récit premier à l'histoire de Mme de la Pommeraye qui, dans le texte original, se trouve enchâssée dans l'histoire du voyage de Jacques et de son maître. Elle y est donc, par le fait même, entrecoupée de digressions et de commentaires du premier narrateur, mais aussi des personnages en présence à ce premier niveau de narration. Le dialogisme diderotien se perd à cause de cette opération de sorte que Buck déplore, pour conclure son étude, la censure schillérienne des libertés esthétiques et morales de l'original (1990, 253). Extraite du dialogue philosophique qu'elle alimente dans le roman de Diderot, la nouvelle paraît sans doute mieux unifiée et plus divertissante pour les lecteurs que Schiller a en tête.

Mais qu'en est-il des portraits et des tableaux au moyen desquels Diderot met en scène les corps de ses personnages? La traduction les rend-elle fidèlement? Rien ne manque, en effet, à ce chapitre, si ce n'est que l'expression est parfois plus dramatique. Si l'amoureux de Diderot est «triste», «mélancolique » et «défait» (Diderot 1962, 630), il se trouve chez Schiller «schwermutsvoll und zugerichtet wie eine Leiche " (dépressif et semblable à un cadavre; 1975e, 200) et le narrateur de préciser que Mme de la Pommeraye en est effrayée, ce qui n'est pas le cas dans le texte original. À une autre occasion, elle retrouve cet amoureux «étendu dans un fauteuil, les yeux fermés, et absorbé dans la plus profonde rêverie » $(1975 \mathrm{e}, 641)$, tandis que dans la version allemande il se trouve «mit geschlossenen Augen, in der schrecklichsten Erstarrung auf dem Sofa ausgestreckt » (étendu les yeux fermés et terriblement raidi sur le sofa; 1975e, 210). De toute évidence, Schiller pousse la somatisation du chagrin à un degré que Diderot pratique lorsqu'il met en scène des gens du peuple, comme dans le conte Les deux amis de Bourbonne, par exemple, mais qu'il évite ici par respect de la décence qu'observent les milieux aristocratiques. Nous avons affaire ici à la marquise de la Pommeraye et au marquis des Arcis dont le propre doit être de maîtriser leurs émotions. Si Schiller passe pour un romantique dans la perception des Français, c'est entre autres à cause de la dramatisation du sentiment dont il reproche la lourdeur à Richardson, mais dont il use néanmoins lui-même. Buck remarque la même tendance au pathos et à l'hyperbole dans les dialogues de la traduction schillérienne (1990, 250-251). Ce n'est donc pas sans raison que Schiller se donne pour modèle l'élégante sobriété de la description diderotienne.

À l'instar de Mme de Merteuil, l'hérö̈ne des Liaisons dangereuses de Laclos, roman épistolaire dont le succès était récent en 1785, Mme de la 
Pommeraye maîtrise ses émotions et peut donc manipuler celles des autres. Dès le début du récit, elle saisit fort bien les signes du désintérêt de son amant croqués de la façon suivante: "Wenn er kam, murmelte er eins, zwei Worte, streckte sich im Sofa, ergriff etwa diese oder jene Broschüre, warf sie weg, schäkerte mit ihrem Hund oder schlief zuletzt gar ein » (Quand il venait, il grommelait un ou deux mots, s'étendait sur le sofa, prenait au hasard une brochure, la jetait, jouait avec le chien et finissait même par s'endormir; Schiller 1975e, 184).

Tandis que l'impatience et la nervosité du marquis des Arcis, nommé simplement $\mathrm{A}^{* * *}$ par Schiller, donnent lieu à plusieurs scènes muettes où on le voit se mouvoir de façon désordonnée, Mme de la Pommeraye se comporte en reine et donne la comédie au marquis. Elle nomme l'inconstance du cœur en feignant ne plus aimer elle-même et en éprouver du chagrin. Les tableaux qui montrent son affliction ne mentent pas, car elle souffre, mais par ses discours elle attribue sa consternation ( «hier drückte die Dame beide Hände vors Gesicht, ließ den Kopf hängen » (1975e, 185)) et ses pleurs (hier drehte sich Frau von $\mathrm{P}^{* * *}$ im Sofa herum und fing laut an zu weinen» $(1975 \mathrm{e}, 186))$ à des scrupules inventés. Curieusement, cet aveu trompeur de manque d'amour entraîne de la part du marquis, heureux de se croire désengagé, une déclaration dont la gestualité respecte les usages de la déclaration d'amour aristocratique. Il se jette à ses pieds (1975e, 186), lui baise les mains $(1975 \mathrm{e}, 187)$ et ne la quitte pas sans l'embrasser (1975e, 188). Ayant gagné par sa feinte la confiance du marquis, Mme de la Pommeraye va pouvoir mener le jeu et conduira le marquis libertin jusqu'au mariage avec une soi-disant dévote qu'elle arrache elle-même à la prostitution pour déclasser son amant infidèle. Tout n'est alors plus que pantomime artificieuse du côté des femmes, tandis que le marquis vit pour de bon une histoire d'amour. En synthétisant la narration complexe de Diderot, Schiller rend cette circonstance parfaitement explicite:

Die Marquisin war schelmisch genug, ihn jeden Fortschritt seiner Leidenschaft bemerken zu lassen, und unter Vorwand, ihn zurückzuschröcken, gewöhnte sie ihn unvermerkt an den verzweifelten Ausgang dieses Romans, den sie ihm bereitet hatte.

La marquise était assez fourbe pour lui faire remarquer tous les progrès de sa passion et, sous prétexte de le dissuader, elle l'habitua peu à peu à l'issue désespérée du roman dans lequel elle l'avait précipité. (Schiller 1975e, 203)

Dans cette histoire, le corps romanesque n'est donc pas celui de la marquise dont le visage n'apparaît qu'au moment où elle va goûter sa vengeance. 
Alors que Diderot n'évoque que l'indignation qui s'y peint «dans toute sa force» $(1962,645)$, Schiller accentue l'aspect démoniaque du personnage qu'il désapprouve: "Man empfing ihn [A**] mit einem Gesicht, worauf Schadenfreude und Entrüstung mit schrecklichen Farben sich malten » (On l'accueillit avec un visage sur lequel le malin plaisir et l'indignation se peignaient en teintes terribles; Schiller 1975e, 212). L'angélisme est réservé à Mlle Duquênoi, belle comme un ange mais sans talents pour séduire des hommes blasés et sans goût pour le métier de prostituée auquel l'indigence de sa mère l'a réduite. Après une première rencontre arrangée à la promenade, Mme de la Pommeraye pousse et aide le marquis à brosser son portrait:

Nun - und was halten Sie von ihr?

Es ist der Kopf einer Mutter Gottes von Raphael, auf den Leib seiner Galathee gestellt - O, und die unaussprechlich melodische Stimme Und die Bescheidenheit im Auge!

Und der Anstand, die Grazie in jeder Gebärde!

Puis - qu'en pensez-vous? C'est la tête d'une vierge de Raphaël déposée sur le corps de sa Galatée - Oh, et quelle voix mélodieuse - Et, l'humilité dans le regard! Et la décence, la grâce de chacun de ses gestes! (Schiller 1975e, 197)

Lidéalisation de la belle prend appui sur la peinture italienne tout comme ce sera le cas lorsque le prince du "Geisterseher " tombera amoureux, frappé par la beauté d'une jeune fille qui prie dans une église à Venise. Il conclut la description enthousiaste qu'il en fait en évoquant le même topos que le personnage de Diderot:

Können Sie sich die Madonna unseres Florentiners zurückrufen? Hier war sie ganz, ganz bis auf die unregelmäßigen Eigenheiten, die ich an jenem Bilde so anziehend, so unwiderstehlich fand.

Vous rappelez-vous la Vierge de notre Florentin? - C'était elle entièrement, jusqu'aux particularités irrégulières que je trouvais si attirantes, si irrésistibles dans ce tableau. (Schiller 1975e, 132)

Le cadre religieux propice à la contemplation, voire à l'adoration de l'élue est un topos voisin qu'on retrouve également dans "Mme de la Pommeraye » et dans le "Geisterseher». Que ne ferait A*** pour voir Mlle Duquênoi! «[F]ünfzehn Tage hat mich keine Messe vermißt - O welche Gestalt, meine Freundin! Wie reizend! Wie unaussprechlich schön!» (Pendant quinze jours, je n'ai pas manqué une seule messe - Oh, quelle figure, mon amie! Quels charmes! Quelle beauté indicible!; Schiller 1975e, 201). L'expérience mystique du prince est plus complète: 
Ich stand [...] in ihrem Anblick verloren. Sie bemerkte mich nicht [...] so ganz war sie in ihrer Andacht vertieft. Sie betete zu ihrer Gottheit, und ich betete zu ihr - Ja, ich betete sie an - Alle diese Bilder der Heiligen, diese Altäre, diese brennenden Kerzen hatten mich nicht daran erinnert; jetzt zum erstenmal ergriff michs, als ob ich in einem Heiligtum wäre.

J'étais perdu dans la contemplation de sa personne. Elle ne me remarquait pas [...] tant elle était plongée dans son recueillement. Elle priait sa divinité et moi, je la priais elle — je l'adorais — Toutes les images des Saints, les autels, les cierges allumés ne m’y avaient pas fait penser; mais maintenant pour la première fois, je fus saisi comme si je me trouvais dans un sanctuaire. (Schiller 1975e, 133)

Le prince visionnaire est beaucoup plus prolixe que le marquis libertin, mais, de part et d'autre, on rehausse l'image de la belle en l'associant à l'art et à la religion.

La sociabilité religieuse est tout aussi codée que la sociabilité aristocratique. Si d'anciennes prostituées peuvent passer du jour au lendemain pour des dévotes, c'est qu'elles acceptent de se comporter selon des règles que leur dicte Mme de la Pommeraye. Celles-ci concernent leurs fréquentations, leurs vêtements, leurs activités, leurs lectures, leurs sorties, leur alimentation et même l'orientation de leurs regards: " [s]chlagen Sie die Augen jederzeit sittsam zu Boden. In der Kirche sehen Sie nirgens hin als auf Gott » (baissez en tout temps les yeux avec décence. À l'église, ne portez vos regards que sur Dieu; Schiller 1975e, 191). Le confesseur de Mlle Duquênoi, qui tâchera d'ailleurs de la dévoyer, n'en lève pas moins les yeux au ciel (1975e, 207), comme il se doit. Il lui remettra ensuite une lettre du marquis, un topos qu'on retrouve également de façon plus élaborée dans le "Geisterseher» (1975e, 148). Mais Schiller ne s'attaque pas avec autant de virulence que Diderot aux abbés corrompus et saute, entre autres, les propos dépréciateurs que la mère d'Aisnon (alias Duquênoi) leur consacre dans la version originale (Diderot 1962, 615). Cette mère se laisserait d'ailleurs tout aussi volontiers acheter que le malheureux confesseur. Comment refuser la cassette pleine de bijoux et les offres d'établissement toujours plus avantageuses que le marquis lui fait parvenir? Mais Mme de la Pommeraye est intraitable. C'est elle la reine devant qui tout le monde s'incline. Le marquis se jette à ses pieds (Schiller 1975e, 186, 205), mais les d'Aisnon aussi (1975e, 209). Après le mariage, cependant, ce geste de soumission traditionnel, éclairant des relations de pouvoir, se déplace. Les 
scènes se succèdent montrant l'héroïne aux pieds d'un mari dont la fureur fait peu à peu place à la résignation, puis éventuellement au bonheur.

Le parcours affectif, qui permet au gentilhomme trompé et offensé de revenir de sa surprise et de sa fureur pour renouer avec son histoire d'amour, assumer sa mésalliance et la transformer en un bonheur privé, est entièrement extériorisé et traduit en mouvements et en déplacements des corps. Un enchaînement de tableaux et de scènes quasiment muettes prépare le moment de la réconciliation du marquis avec la nouvelle marquise. Ces dernières tirades ont un impact idéologique digne du parti des philosophes. On y reconnaît la verve de l'inventeur du drame bourgeois qui combat les préjugés et interroge la vérité de la nature pour fonder un bonheur domestique accessible à tous. Le corps n'est pas censé mentir et maintenant que Mme de la Pommeraye, qui tirait toutes les ficelles de la farce ( «des Possenspiels ", Schiller 1975e, 198), est sortie du jeu, il est possible d'observer la Nature.

Selon son tempérament, Schiller accentue le drame. Ainsi le marquis s'effondre («zu Boden sank», 1975e, 213) en apprenant la vérité sur le passé de sa femme. Ceci n'est pas le cas dans l'original. Puis, sa femme s'évanouit ou demeure impuissante au premier mot de colère qu'il lui adresse ( "ohnmächtig stürzte sie auf ihr Angesicht », 1975e, 213). L'ajout de l'adverbe "ohnmächtig» (évanouie) étonne, puisqu'elle prend la parole tout de suite après sa chute. Plus loin, cependant, Schiller retient la violence du geste du marquis ( "war im Begriff, ihr einen grausamen Schlag zu geben »; 1975e, 214) alors que Diderot dit carrément: «la poussa durement » (1962, 646). Au fond, il n'est pas étonnant que le traducteur modifie la gestualité inscrite dans le texte original en fonction de ce qui lui semble naturel selon sa propre sensibilité. Ceci dit, Schiller suit de très près la longue pantomime qui extériorise la peine qu'éprouve la ci-devant d'Aisnon. Ce qui est pour Diderot «la figure du désespoir» $(1962,646)$ sera pour lui «das traurigste Bild der Reue, des Schmerzens, der Verzweiflung» (le plus triste tableau du repentir, de la douleur et du désespoir; Schiller 1975e, 214). Ainsi, il retient, entre autres, tous les détails du tableau de l'imploration du mari, hormis le geste des «bras portés de son côté » (Diderot 1962, 647):

An der Schwelle sank sie auf die Knie. Er befahl ihr aufzustehen. Sie stand nicht auf, sondern wälzte sich in dieser Stellung näher zu ihm hin. Alle ihre Glieder zitterten. Ihre Haare waren losgebunden. Ihr Leib hing zur Erde, ihr Kopf war emporgerichtet und ihre Augen, die von Tränen flossen, begegneten den seinigen. 
Sur le seuil elle se jeta à genoux. Il lui ordonna de se lever. Elle ne se leva pas, mais se traina dans cette position jusqu'à lui. Tous ses membres tremblaient. Ses cheveux étaient défaits. Son corps était à terre, mais elle tenait la tête et les yeux levés et son regard, plein de larmes, rencontra le sien. (Schiller 1975e, 215)

Le marquis ne tardera pas à accorder son pardon à cette malheureuse et à affirmer qu'il honore en elle sa femme. Elle n'ose cependant pas y croire, embrasse son mari dans un bref élan de tendresse, puis se jette de nouveau à ses pieds ( «willens, seine Füße zu küssen "; 1975e, 216). L'image fige le couple dans la pose de l'homme miséricordieux et de la femme entièrement soumise. Dès lors, le marquis a quelques raisons de croire qu'il sera heureux comme un roi, dans ses terres, loin du monde. Le voilà à son tour vengé de la fierté de Mme de la Pommeraye et en bonne position pour jouir du système patriarcal cautionné par les élans de la Nature ou du cœur tels que les donnent à voir les mouvements des corps dans cette scène pathétique qui clôt la nouvelle. Et Schiller d'ajouter que les agissements de Mme de la Pommeraye n'étaient pas naturels ( «diese unnatürliche Tat»; 1975e, 218), tandis que le narrateur de Jacques le fataliste se souvient du libertinage du marquis des Arcis qui n'était pas honnête. Selon la sagesse de ce narrateur, la loi devrait, donnant raison à Mme de la Pommeraye, condamner le libertin aux courtisanes: "l'homme commun aux femmes communes" (Diderot 1962, 652). Diderot disserte sur les hommes en général, tandis que Schiller propose à ses lecteurs une histoire hors du commun pouvant susciter l'admiration. Le jugement moral qui s'ensuit condamne l'intrigante et retient les beaux gestes des amants réconciliés en dépit du préjugé de classe. Le sublime schillérien pourrait se trouver du côté du marquis car l'effort raisonné de sa volonté lui permet de surmonter sa douleur.

\section{Histoires allemandes}

Après cette adaptation d'une nouvelle de Diderot, Schiller publie ses propres nouvelles dans les cahiers subséquents de sa revue Thalia: en 1786, «Verbrecher aus Infamie, eine wahre Geschichte »; puis en 1787, 1788 et 1789, cinq épisodes du "Geisterseher ». "Spiel des Schicksals » paraît également en 1789, mais dans une autre revue. À vrai dire, seul le "Geisterseher» relève vraiment de la fiction et constitue un fragment de roman. Les épisodes ont cependant leur autonomie et leur enchaînement sera réaménagé ensuite pour publication sous forme de livre. On peut voir l'ensemble de nouvelles publiées entre 1786 et 1789 comme un développement de ce qui 
avait été posé dans la première nouvelle de 1782. Schiller proposait alors deux destins possibles pour les Allemands, soit celui de rester au pays, soit celui de s'expatrier. Cette alternative est bien connue. C'est celle de Sindbad le marin et Sindbad le portier, archétypes du voyageur et du sédentaire, dont Bruno Bettelheim a montré qu'ils incarnaient le principe de plaisir et le principe de réalité (1977, 83-86). Schiller illustre donc, d'une part, deux destinées allemandes qui se jouent au pays: celle de Christian Wolf, le Criminel par infamie qui épouse la carrière de Friedrich Schwan (17291760), brigand réel dont l'histoire fut également publiée par Jakob Friedrich Abel; et, dans "Spiel des Schicksals ", celle de Aloysius von G*** dont le modèle dans la vie réelle était le général Philipp Friedrich von Rieger, parrain de Schiller, décédé en 1782. D'autre part, Schiller goûtera sans grand enthousiasme au principe de plaisir en envoyant un prince allemand fictif à Venise et en l'exposant à plusieurs aventures et histoires italiennes dans le «Geisterseher».

L'étude de la plasticité des corps et de leur interaction dans ces nouvelles révèle du côté allemand l'inévitable exil intérieur du héros. Qu'il soit issu du peuple, comme Wolf, ou de la noblesse, comme Aloysius von $\mathrm{G}^{* * *}$, que ses premières années soient difficiles ou au contraire glorieuses, il est mis au ban de la société et fera l'expérience de la prison. Les portraits de ces héros sont diamétralement opposés. Wolf n'a pas été favorisé par la nature:

Eine kleine unscheinbare Figur, krauses Haar von einer unangenehmen schwärze, eine plattgedrückte Nase und eine geschwollene Oberlippe, welche noch überdies durch den Schlag eines Pferdes aus ihrer Richtung gewichen war, gaben seinem Anblick eine Widrigkeit, welche alle Weiber von ihm zurückscheuchte und dem Witz seiner Kameraden eine reichliche Nahrung darbot.

Une petite taille insignifiante, des cheveux crépus d'un noir désagréable, un nez aplati et une lèvre supérieure gonflée qui en outre, à cause d'un coup de sabot de cheval, avait dévié de sa direction, donnaient à son aspect un air rébarbatif qui faisait fuir toutes les femmes et alimentait largement les railleries de ses camarades. (Schiller 1975e, 16; trad. R. Radrizzani, p. 16)

De son côté, Aloysius à été richement doté:

Den Wert [seiner] Eigenschaften erhöhte eine empfehlende Gestalt, das volle Bild blühender Gesundheit und herkulischer Stärke, durch das beredte Spiel eines regen Geistes beseelt; im Blick, Gang und Wesen eine anerschaffene natürliche Majestät, durch eine edle Bescheidenheit gemildert. 
La valeur de ses qualités était rehaussée par une figure recommandable, l'image même d'une santé resplendissante et d'une force herculéenne animée par le jeu éloquent d'un esprit vif; dans le regard, la démarche et l'être tout entier, une majesté naturelle, adoucie par une noble modestie. (Schiller 1975e, 36)

Les prémisses de la déchéance et celles de la gloire étant posées, chaque personnage suit sa destinée jusqu'au moment où un événement irréparable se produit. Dans les deux cas, il s'agit de l'interaction avec un rival. Le loup (Wolf) tuera son chasseur (Jäger Robert), tandis que le favori du prince, devenu ministre, sera publiquement dégradé par un nouveau favori italien du nom de Martinengo. Ces moments dramatiques donnent lieu à une mise en présence des corps qui permet de contraster la technique du tableau, révélant l'intériorité, de la technique de la scène révélant la sociabilité des personnages. Le cadre de ces événements distingue bien leurs qualités respectives puisque Wolf se cache dans la forêt, tandis qu'Aloysius rejoint le cercle de ses habitués.

La scène de la destitution d'Aloysius se situe au milieu du récit. Martinengo exécute cette opération au nom du prince. Ses paroles ne sont pas rendues en style direct, mais son changement d'attitude, son pas et la façon dont il brise l'épée du héros sont minutieusement détaillés. Le corps du ministre n'est pas par contre évoqué, mis à part son regard consterné. Ce sont ses attributs qui comptent. Épée, croix, épaulettes, parements, cordon, plumet, toutes ces distinctions qui donnent à voir son mérite et son pouvoir lui seront arrachées. Ces gestes le privent symboliquement de son statut et mettent en place sa mort sociale avant même qu'il ne soit conduit en prison. Il reste constant dans le malheur grâce à sa force d'âme, mais le corps social assemblé autour de lui offre alors le tableau de la souffrance causée par ce revirement du destin:

Mit bleichen Gesichtern, mit klopfendem Herzen und in totenähnlicher Erstarrung steht die erschrockene Menge im Kreis um ihn herum.

La foule se tient épouvantée autour de lui, les visages pâles, les cœurs battants, raide comme dans la mort. (Schiller 1975e, 42)

Seize mois plus tard, le brillant ministre n'est plus qu'un monstre au fond de son cachot:

Ein blasses totenähnliches Gerippe, alle Farbe des Lebens aus einem Angesicht verschwunden, in welches Gram und Verzweiflung tiefe Furchen gerissen hatten, Bart un Nägel durch eine so lange Vernachlässigung bis zum Scheußlichen gewachsen. 
Un squelette pâle comme la mort, toutes les couleurs de la vie ayant quitté un visage dans lequel la peine et le désespoir avaient creusé des rides, tandis que la barbe et les ongles avaient poussé de façon répugnante. (Schiller 1975e, 45)

Les réparations qui lui seront offertes par la suite ne parviendront plus à refaire de cet être dégradé un homme bon et Aloysius mourra en maltraitant les prisonniers dont le prince lui aura confié la garde en le nommant commandant de sa forteresse. Ainsi, une perfidie en entraîne d'autres et la dureté se perpétue dans la société allemande.

La critique a surtout commenté l'engagement de Schiller concernant la psychologie des malfaiteurs blessés par la société en étudiant «Der Verbrecher aus verlorener Ehre ». S'intéressant à la nature de ce type de narration engagée, Achim Aurnhammer offre un survol raisonné de la critique consacrée à cette nouvelle (1990, 255-256) et s'arrête, entre autres, à l'alternance du résumé narratif à la troisième personne et de l'élaboration scénique offerte dans la perspective du personnage de Christian Wolf. Il remarque alors la double finalité de la narration engagée de Schiller qui touche le cœur et l'esprit («[das Erzählen] appelliert in der Perspektive des Ich-Erzählers an das Gefühl und im Bericht des Er-Erzählers an den Verstand »; Aurnhammer 1990, 261). La scène capitale du meurtre de Jäger Robert est narrée à la première personne et serre de près les émotions du meurtrier. Wolf a déjà fait trois fois de la prison parce que Jäger Robert a dénoncé son braconnage. À la fin de sa troisième peine son aspect est aussi répugnant que celui d'Aloysius au fond de sa prison: "der Bart, den ich noch von der Festung mitbrachte [entstellte] meine Gesichtszüge bis zum Gräßlichen» la barbe que je rapportais de la forteresse, me défigurait affreusement les traits (Schiller 1975e, 19; trad R. Radrizzani, p. 24). Rejeté par un enfant, il se demande si on voit sur son front qu'il ne peut plus aimer personne. Puis, sa dureté est confirmée par la façon dont il rejette lui-même son ancienne amie Johanne, parce qu'il voit qu'elle est marquée par la petite vérole. Retombé dans le braconnage, il tient subitement son pire ennemi, Jäger Robert, en joue au même moment que le chevreuil qu'il poursuivait:

Der Arm zitterte mir, da ich meiner Flinte die schreckliche Wahl erlaubte - meine Zähne schlugen zusammen wie im Fieberfrost, und der Odem sperrte sich erstickend in meiner Lunge. [...] Lange stand ich vor dem Toten, ein helles Gelächter machte mir Luft.

Mon bras tremblait, comme je permettais à mon fusil ce choix terrible - mes dents claquaient comme dans un frisson de fièvre et la respiration se bloquait, étouffante dans mes poumons [...] 
Longtemps, je restai muet devant le mort, un rire clair enfin me libéra. (Schiller 1975e, 22; trad. R. Radrizzani, p. 29)

L'irréparable a été commis non pas de sang-froid, mais dans une sorte de crise physique dont Wolf ne se réveille qu'à l'approche d'éventuels témoins. Sa fuite le jette alors dans les bras d'un brigand à la bande duquel il s'associera. Tandis qu'Aloysius souffre son destin, brutalisé par Martinengo et ses acolytes, Wolf forge le sien mû par un besoin de vengeance dont l'impact secoue également son corps.

Il y aurait encore bien des choses à dire sur le corps puissant et laid du brigand et sur l'interaction de Wolf avec la bande dont il deviendra le chef, comme Karl Moor dans la pièce des Brigands. Et, comme ce dernier, il finira par se rendre à la justice, mais non sans avoir été repéré par un fameux physionomiste qui, par métier, scrute les visages des vagabonds aux portes d'une ville depuis quarante ans: «der Falkenblick dieses Spürers verfehlte auch hier seinen Mann nicht» (l'œil de faucon de ce limier, une fois de plus, ne manqua pas son homme; Schiller 1975e, 32; trad. R. Radrizzani, p. 51). On sait, en effet, que les gardes du corps et sans doute aussi les gardiens des villes sont les plus habiles à repérer les suspects dont la mine et le comportement manquent d'unité et de cohérence. C'est bien ce que Schiller donne à voir dans son dernier portrait de Wolf:

Der hagere Klepper, den er ritt, und die burleske Wahl seiner Keidungsstücke, wobei wahrscheinlich weniger sein Geschmack als die Chronologie seiner Entwendungen zu Rat gezogen war, kontrastierte seltsam genug mit dem Gesicht, worauf so viele wütende Affekte, gleich den verstümmelten Leichen auf einem Walplatz, verbreitet lagen.

Le maigre canasson qu'il montait et le choix burlesque de ses vêtements, auquel avait présidé moins son goût que la chronologie de ses rapines, contrastaient assez étrangement avec un visage où tant de passions violentes s'étalaient tels des cadavres mutilés sur un champ de bataille. (Schiller 1975e, 31; trad. R. Radrizzani, p. 51)

De toute évidence, le corps et le visage du personnage schillérien constituent un langage socio-affectif que le narrateur peut se plaire à expliquer lui-même par des remarques et des comparaisons colorées, voire lugubres! En l'occurrence, le lecteur a déjà été informé de l'exécution du héros (1975e, 15 ) et doit pouvoir déchiffrer la souffrance qui l'a poussé à risquer la peine capitale pour tenter sa réinsertion dans le corps social et retrouver la liberté de se nommer, comme il le fera à la toute fin du récit en se dénonçant luimême alors qu'il se sent en confiance. Il implore la clémence des autorités, 
mais celles-ci ne verseront pas une larme sur lui, quoiqu'il le demande (Schiller 1975e, 35). Son aveu fatal est admirable et met en cause une justice sans nuances.

Le bandit attendri et l'ancien favori endurci se font face dans les deux histoires allemandes de Schiller. Cette complémentarité qui forme un tout est en quelque sorte scellée par la symbolique de l'échelle qui se retrouve de part et d'autre. Wolf emprunte une échelle pour descendre dans le repère des brigands après avoir rêvé de la retirer pour échapper à l'enfer (Schiller 1975e, 27); Aloysius gravit les échelons qui mènent au pouvoir en recherchant l'amitié du prince, mais, arrivé à ses fins, il laisse tomber l'échelle derrière lui $(1975 \mathrm{e}, 39)$ sans penser qu'il faut toujours assurer ses arrières quand on flatte le prince dans une société de cour. Les portraits, les tableaux et les scènes qui font voir le corps des héros déchus montrent que l'exclusion est intenable, enlaidit et engendre le crime et la dureté. Même si le comportement inhumain d'Aloysius s'explique par son vécu, sa dureté semble inacceptable parce qu'il exerce du pouvoir. Le cas de Wolf est différent. Malgré son meurtre, il est moins sûr que le lecteur puisse le condamner car son humanité s'aperçoit dans les tableaux qui révèlent sa souffrance. L'autopsie du vice annoncée par le narrateur (Schiller 1975b, 15) s'attache en fait au corps sensible du supplicié. S'il y a une moralité du geste commune aux héros des nouvelles analysées jusqu'ici, elle réside dans un renoncement volontaire qui assure le bien d'une communauté. Le plus jeune frère de la première nouvelle renonce à son amour pour le bien de sa famille, le marquis des Arcis renonce au monde pour sauver son mariage, Wolf renonce à la protection de l'incognito pour quitter la contre-société de brigands. Quant à Aloysius, il montre bien de la constance dans le malheur et fait aussi dans ce sens un effort sur lui-même, mais il n'a pas le choix et c'est plutôt l'Italien Martinengo, son agresseur, qui, par son geste, prend tout le monde par surprise.

\section{Histoires italiennes}

Les rapports tendus entre Allemands et Italiens sont explorés plus avant dans les cinq épisodes du "Geisterseher ", texte alimentaire, paru comme un roman-feuilleton dans plusieurs numéros de la revue Thalia dans le but d'en élargir le lectorat. Ce roman, dont on connaît le plan, est resté inachevé et ne répond donc pas à l'exigence d'unité chère à Schiller et parfaitement maintenue dans la traduction et les histoires allemandes vues jusqu'ici. Nous avons déjà observé que la reprise occasionnelle de quelques topoï descriptifs des amours romanesques instaure une certaine continuité for- 
melle entre ce roman et l'histoire de Mme de la Pommeraye. Le lien le plus marquant est cependant le fait que, de part et d'autre, le héros est manipulé par d'autres personnages. Ceci dit, les stratégies narratives ne se ressemblent pas, puisque le jeu de Mme de la Pommeraye est transparent, tandis que celui de l'Arménien qui obsède le prince visionnaire est tout à fait caché. Le jeu de cache-cache est d'ailleurs initié par le prince allemand lui-même, car il veut vivre à Venise incognito, sort masqué et remarque aussitôt qu'il est suivi par un autre masque. "Die Maske war ein Armenier und ging allein » (Schiller 1975e, 50). Cette circonstance prive le lecteur des portraits du prince et de l'Arménien. Et quand celui-ci réapparaît en officier russe, son visage demeure insaisissable:

Nie in meinem Leben sah ich so viele Züge und so wenig Charakter, so viel anlockendes Wohlwollen mit so viel zurückstoßendem Frost in einem Menschengesichte beisammen wohnen. [...] Nichts war übrig als der stille, durchdringende Blick eines vollendeten Menschekenners, der jedes Auge verscheuchte, worauf es traf. Jamais de ma vie je n'avais vu sur un seul visage humain tant de traits réunis et si peu de caractère, tant de bienveillance attirante réunie à tant de glace repoussante. [...] Il ne restait plus que le regard tranquille et pénétrant d'un connaisseur d'hommes accompli, qui faisait fuir tous les regards qu'il rencontrait. (Schiller 1975e, 56)

Ce portrait brouille les indices usuels permettant au lecteur de saisir l'identité d'un personnage dans les traits de son visage et ses affects dans ses mimiques. Tout est indécis, sauf le regard perçant du physionomiste. On a sans doute affaire à un intrigant. Ce personnage énigmatique ou insondable ( «bei uns kennt man ihn nur unter dem Namen des Unergründlichen»; Schiller 1975e, 76-77) est aussi bien un officier de l'Inquisition étatique (1975e, 69) qu'un être surnaturel quittant le monde des vivants pour une heure toutes les nuits. Son visage s'assombrit alors et son corps se raidit (1975e, 77-78). Les histoires fabuleuses ne manquent pas à son sujet, comme celle que raconte le Sicilien au sujet du Marchese del M**nte. À vrai dire, il est parfaitement conséquent que des esprits échappent aux déterminations corporelles et psychologiques que nous avons observées dans les histoires vraies situées en Allemagne.

À Venise, le prince allemand perd peu à peu ses repères. Il est pris en charge par un personnel mystérieux très inquiétant, si l'on pense à l'Arménien et au Sicilien, mais aussi très rassurant, si l'on pense à Biondello, le nouveau secrétaire italien du prince, dont la physionomie plaît (Schiller 1975e, 71), et l'efficacité convient, car il voit tout et règle tout «als wenn er tausend 
Augen hätte, tausend Hände in Bewegung setzen könnte » (comme s'il pouvait mettre en mouvement mille yeux et mille mains; Schiller 1975e, 115). Ce débordement, cette exagération marquent bien l'altérité culturelle des Italiens. Cette altérité est aussi religieuse. Comme c'est le cas dans Don Carlos, le moteur de l'intrigue du «Geisterseher » se trouve dans l'opposition entre protestants et catholiques. Tout le personnel mystérieux qui entoure le héros semble être à la solde d'un richissime cardinal et semble être chargé de ramener le prince protestant dans le giron de l'Église catholique. Cette circonstance n'était pas sans lien avec des rumeurs voulant à l'époque que certains jésuites cherchassent à convertir le prince héritier protestant du Wurtemberg au catholicisme. On voit le piquant que cette clé pouvait donner au roman-feuilleton.

Dans le récit, le prince a beau comprendre par quelles astuces il est joué, il n'en sera pas moins mené jusqu'à l'apostasie dans l'idée de sa famille allemande (Schiller 1975e, 155). Son roman d'amour ne le sauvera pas non plus, car l'Allemande expatriée, qu'il prend pour une belle Grecque, mourra empoisonnée dans les plus brefs délais. Cette façon de couper court au développement du roman montre que Schiller n'a pas l'intention de sacrifier longtemps au principe de plaisir en conduisant ses personnages allemands sur les voies d'un exil troublant et aventureux. Au lieu d'expliquer des faits, comme les nouvelles allemandes, les histoires italiennes se développent sur un fond de complot. Cette stratégie narrative toujours ouverte à de nouvelles illusions qui seront suivies de nouvelles démystifications, comme le montre Monika Schmitz-Emans (1996), ne permet pas de boucler un sujet, ni de saisir la vérité d'une destinée de façon cohérente comme le veut la poétique de Schiller.

Mais en le dévoyant, son besoin d'argent a aussi permis à Schiller de s'abandonner à une muse plus échevelée qui le pousse davantage vers les profondeurs de l'inconscient inconciliables avec sa recherche du sublime volontaire et des gestes admirables. Il serait intéressant dans un autre contexte de scruter plus à fond la représentation des corps du personnel de roman mystérieux du "Geisterseher». Il est recouvert de masques qu'il s'agit de faire tomber pour découvrir des corps parlant peut-être de la peur et de la mort que les soldats de Wallenstein, stimulés par l'exemple de leur général, tiennent à distance en chantant:

Der dem Tod ins Angesicht schauen kann

Der Soldat allein ist der freie Mann

Pouvant voir la mort en face, seul le soldat est un homme libre.

(Schiller 1975b, 309) 
Il est vrai que la présence du corps, si révélatrice de l'humanité des êtres entiers et vivants, indispensable aussi à l'exercice du charisme, est plus immédiatement convaincante au théâtre que dans l'écriture romanesque, même si celle-ci est très attentive à l'identité des traits, aux marques des passions et aux mouvements interpersonnels qui caractérisent les personnages en faisant voir leur corps. La question de savoir si Schiller, dramaturge, exige trop du corps de ses personnages, comme le veut Matthew Bell $(1999,197)$ selon une étude stimulante nourrie de psychologie et de psychanalyse, dépasse bien sûr le propos de cet essai. Mais en suivant dans les nouvelles de Schiller, apprenti romancier, la distribution et la configuration des portraits, tableaux et scènes qui comptent à l'époque des Lumières européennes parmi les stratégies courantes de la présentation du corps sensible dans le roman, il a été possible de montrer la particularité des nouvelles allemandes. Tout en s'appuyant sur des faits véritables, elles développent leur propre unité et leur vérité poétique en construisant une cohésion juste et belle entre le corps et l'âme, indispensable à une morale des gestes posés par les personnages. Les gestes admirables qui en découlent mettent alors en cause de façon crédible les intrigues courtisanes et les duretés arbitraires de l'absolutisme. Dans ce sens, les œuvres elles-mêmes sont des gestes engagés qui frappent encore aujourd'hui.

\section{Références}

ABEL, J. F. (1787), Sammlung und Erklährung merkwürdiger Erscheinungen aus dem menschlichen Leben, t. 2., Francfort/Leipzig.

Aurnhammer, A. (1990), «Engagiertes Erzählen. Der Verbrecher aus verlorener Ehre ", dans A. Aurnhammer, K. MANGer et F. STRACK, dir., Schiller und die höfische Welt, Tübingen, Niemeyer Verlag, p. 254-270.

BELL, M. (1999), "The Revenge of the "untere Seelenvermögen" in Schiller's Plays ", German Life and Letters, 52/2, p. 197-210.

BetTelheim, B. (1977), The Uses of Enchantment. The Meaning and Importance of Fairy Tales, New York/Toronto, Random House.

BuCK, S. (1990), «Nachdichtung oder Übersetzung? Diderots Mme-de-laPommeraye-Episode in der Bearbeitung Schillers ", dans A. AURNHAMMER, K. MANGER et F. STRACK, dir., Schiller und die höfische Welt, Tübingen, Niemeyer Verlag, p. 247-253.

Descartes, R. (1953), CEuvres et lettres, éd. par A. Bridoux, Paris, Gallimard. 
Diderot, D. (1962), Jacques le fataliste. CEuvres romanesques, éd. par H. Bénac, Paris, Garnier, p. 491-780. (1996), Euvres, t. IV : Esthétique. Théâtre, éd. par L. Versini, Paris, Robert Laffont (Bouquins).

Ebnet, K.-H. (1993), "Einleitung ", dans F. SCHILleR, Der Geisterseher. Sämtliche Erzählungen, éd. par K.-H. Ebnet, Kehl, Swan, p. 9-17.

Goethe-Schiller. Correspondance 1794-1805 (1994a), t. 1, trad. par L. Herr, Paris, Gallimard.

Goethe-Schiller. Correspondance 1794-1805 (1994b), t. 2, trad. par L. Herr, Paris, Gallimard.

Hartmann, P. (1997), Du Sublime. De Boileau à Schiller, Strasbourg, Presses universitaires de Strasbourg.

LAFON, H. (2001), «Le Roman au miroir du dramatique ", Eighteenth-Century Fiction, 13/2-3, p. 437-459.

Moser-Verrey, M. (2001), « Le Langage du corps romanesque des Illustres françaises (1713) à La Sorcière de Verberie (1798) ", Eighteenth-Century Fiction, 13/2-3, p. 349-388.

- (2006), «Infléchir l'histoire? Schiller poète des conjurations ", dans S. Vervacke, É. VAN Der Schueren et T. Belleguic, dir., Les songes de Clio. Fiction et Histoire sous l'Ancien Régime, Québec, Presses de l'Université Laval (Les collections de la République des Lettres), p. 325-339.

PÉJu, P. (1996), «Le théâtre des influences », dans F. SCHILLER, Le visionnaire, trad. par Albert Béguin, Paris, José Corti (Romantique; 58), p. 7-54.

RAdRIZZANI, R. (1990), «Postface», dans F. SCHILleR, Criminel par infamie suivi de J. F. ABEL, Histoire de la vie de Friedrich Schwan, trad. par R. Radrizzani, Paris, José Corti (Romantique; 26), p. 85-122.

Rothmann, K. (1999), Erläuterungen und Dokumente. Friedrich Schiller. Wallenstein, Stuttgart, Reclam.

Schiller, F. (1975a), Sämtliche Werke, t. 1, éd. par G. Fricke et H. G. Göpfert, Munich, Carl Hanser.

(1975b), Sämtliche Werke, t. 2, éd. par G. Fricke et H. G. Göpfert, Munich, Carl Hanser.

(1975c), Sämtliche Werke, t. 3, éd. par G. Fricke et H. G. Göpfert, Munich, Carl Hanser.

- (1975d), Sämtliche Werke, t. 4, éd. par G. Fricke et H. G. Göpfert, Munich, Carl Hanser. 
(1975e), Sämtliche Werke, t. 5, éd. par G. Fricke et H. G. Göpfert, Munich, Carl Hanser.

(1998), Textes esthétiques. Grâce et dignité et autres textes, trad. par N. Briand, Paris, J. Vrin.

SchmiTZ-Emans, M. (1996), "Zwischen Wahrem und Falschem Zauber. Magie und Illusionistik als metapoetische Gleichnisse. Eine Interpretation zu Schillers "Geisterseher” ", Zeitschrift für deutsche Philologie, 115/Supplément, p. 33-43.

StAiger, E. (1964), «Nachwort », dans F. SCHILler, Erzählungen, Francfort, Insel-Verlag, p. 265-284.

WIESE, B. Von (1963), Friedrich Schiller, Stuttgart, J. B. Metzlersche Verlagsbuchhandlung.

\section{Résumé}

Le charisme en question est abordé par le biais de sa représentation dans Le camp de Wallenstein, pièce construisant par l'échange d'opinions le pouvoir extraordinaire d'un leader absent. Ce paradoxe illustre à merveille le travail du poète qui, par ses mots, donne à voir des corps vivants et des gestes admirables. L'analyse des figures du corps parlant porte sur le corpus des nouvelles de Schiller et révèle sa maîtrise de l'écriture sensible en vogue dans l'Europe des Lumières. Elle montre aussi son souci de l'unité classique et sa recherche du beau geste critique des intrigues courtisanes et de l'absolutisme arbitraire.

\section{Abstract}

The charisma called into question is first discussed by the way of its representation in Wallenstein's Camp, a play in which the dialog shapes the special powers of the absent leader. This paradox illustrates the task of the poet to shape his characters, body and soul as well as their memorable gestures with mere words. The analysis of the narrated body language concentrates on Schiller's short stories and reveals his familiarity with Richardson's and Diderots' poetics. It also shows his sense of classical unity and his choice of beautiful gestures as an alternative to the abuses seen in German courts. 